\title{
Crossings and nestings over some Motzkin objects and $q$-Motzkin numbers
}

\author{
Sandrataniaina R. Andriantsoa Paul M. Rakotomamonjy \\ Department of Mathematics and Computer Science \\ Sciences and Technology, PB 906 Antananarivo 101 \\ Madagascar \\ $\{$ andrian.2sandra, rpaulmazoto\}@gmail.com
}

Submitted: Dec 20, 2020; Accepted: Jun 17, 2021; Published: Jul 16, 2021

(C) The authors. Released under the CC BY-ND license (International 4.0).

\begin{abstract}
We examine the enumeration of certain Motzkin objects according to the numbers of crossings and nestings. With respect to continued fractions, we compute and express the distributions of the statistics of the numbers of crossings and nestings over three sets, namely the set of 4321-avoiding involutions, the set of 3412-avoiding involutions, and the set of $(321,3 \overline{1} 42)$-avoiding permutations. To get our results, we exploit the bijection of Biane restricted to the sets of 4321- and 3412-avoiding involutions which was characterized by Barnabei et al. and the bijection between (321,3142)-avoiding permutations and Motzkin paths, presented by Chen et al. . Furthermore, we manipulate the obtained continued fractions to get the recursion formulas for the polynomial distributions of crossings and nestings, and it follows that the results involve two new $q$-Motzkin numbers.
\end{abstract}

Mathematics Subject Classifications: 11A55, 05A19, 05A15,05A05

\section{Introduction and result}

We let $S_{n}$ denote the set of all permutations of $[n]:=\{1, \ldots, n\}$. A permutation $\sigma \in S_{n}$ is an involution whenever $\sigma(\sigma(i))=i$ for all $i \in[n]$, and we write $I_{n}$ to denote the set of all involutions in $S_{n}$. We refer $|\sigma|$ as the length of the permutation $\sigma$.

Suppose that $\sigma \in S_{n}$ and $\tau \in S_{k}$. We say a subsequence $s=\sigma\left(i_{1}\right) \sigma\left(i_{2}\right) \cdots \sigma\left(i_{k}\right)$ is an occurrence of $\tau$ if and only if $s$ and $\tau$ are in the same isomorphic order, i.e. $\sigma\left(i_{x}\right)<\sigma\left(i_{y}\right)$ if and only if $\tau(x)<\tau(y)$. Whenever $\sigma$ contains no occurrence of $\tau$, we say that $\sigma$ avoids the pattern $\tau$ or simply $\sigma$ is $\tau$-avoiding. For any given set of permutations $T$, called set of patterns, we write $S_{n}(T)$ and $I_{n}(T)$ to denote the sets of permutations and involutions, respectively, in $S_{n}$ which avoid every pattern in $T$, and we write $S(T)$ and $I(T)$ to denote the sets of all permutations and involutions, respectively, including the 
empty permutation, which avoid every pattern in $T$. Usually, if $T=\left\{\tau_{1}, \ldots, \tau_{k}\right\}$, we write $S_{n}(T)=S_{n}\left(\tau_{1}, \ldots, \tau_{k}\right)$ and $S(T)=S\left(\tau_{1}, \ldots, \tau_{k}\right)$. For example, the subsequence 5297 of the permutation $\pi=35142987$ is an occurrence of 2143 . We can easily verify that $\pi \in I_{9}(4321)$.

A barred permutation is a permutation such that some of its elements are barred. We let $\bar{S}_{k}$ denote the set of all barred permutations of length $k$. Example: $3 \overline{2} 4 \overline{5} 1$ is a barred permutation in $\bar{S}_{5}$. Whenever $\bar{\tau} \in \bar{S}_{k}$, we let $\tau$ denote the permutation obtained by unbarring $\bar{\tau}$, and $\tau^{\prime}$ denote the reduction of the permutation obtained from $\bar{\tau}$ by removing the barred element (if any). Example: if $\bar{\tau}=3 \overline{2} 4 \overline{5} 1$, we have $\tau=32451$ and $\tau^{\prime}=231$. We say that a permutation $\sigma$ avoids $\bar{\tau}$ if each occurrence of $\tau^{\prime}$ in $\sigma$ (if any) is part of an occurrence of $\tau$ in $\sigma$. For example, a permutation $\sigma$ is 3142-avoiding if and only if, for any occurrence $\sigma(i) \sigma(j) \sigma(k)$ of 321 in $\sigma$, there exists $i<l<j$ such that $\sigma(l)<\sigma(k)$.

A statistic over a given set $E$ is a map $s: E \rightarrow \mathbb{N}$. The polynomial distribution of the statistic $s$ over the $E$ is the polynomial $\sum_{e \in E} q^{s(e)}$. For example, if we let $\mathcal{P}_{n}$ denote the set of all subsets of $[n]$ and $\operatorname{card}(A)$ denote the cardinality of $A$ for any $A \in \mathcal{P}_{n}$, then card is a statistic over $\mathcal{P}_{n}$ and its polynomial distribution is

$$
\sum_{A \in \mathcal{P}_{n}} q^{\operatorname{card}(A)}=(1+q)^{n}
$$

Furthermore, the generating function of the polynomial $\sum_{A \in \mathcal{P}_{n}} q^{\operatorname{card}(A)}$ is

$$
1+\sum_{n \geqslant 1} \sum_{A \in \mathcal{P}_{n}} q^{\operatorname{card}(A)} t^{n}=\frac{1}{1-(1+q) t} .
$$

We will now define some statistics over $S_{n}$. For that, we let $\sigma \in S_{n}$. An excedance (resp. fixed point) of $\sigma$ is an index $i$ such that $\sigma(i)>i$ (resp. $\sigma(i)=i$ ). A crossing (resp. nesting, inversion) of $\sigma$ is a pair of indices $(i, j)$ such that $i<j<\sigma(i)<\sigma(j)$ or $\sigma(i)<\sigma(j) \leqslant i<j$ (resp. $i<j<\sigma(j)<\sigma(i)$ or $\sigma(j)<\sigma(i) \leqslant i<j, i<j$ and $\sigma(j)>\sigma(i))$. We let $\operatorname{exc}(\sigma)$ (resp. $\operatorname{cr}(\sigma), \operatorname{nes}(\sigma), \operatorname{inv}(\sigma))$ denote the number of excedances (resp. crossings, nestings, inversions) of $\sigma$. For example, the permutation $\pi=46298173105 \in S_{10}$ has the following properties: $\operatorname{exc}(\pi)=5, \operatorname{cr}(\pi)=7$, $\operatorname{nes}(\pi)=4$ and $\operatorname{inv}(\pi)=20$. Therefore, exc, cr, nes, and inv are all statistics over $S_{n}$. Médicis and Viennot [17] and Randrianarivony [21] showed that these statistics are related by the following identity:

$$
\operatorname{inv}(\sigma)=\operatorname{exc}(\sigma)+\operatorname{cr}(\sigma)+2 \operatorname{nes}(\sigma), \text { for any } \sigma \in S_{n} .
$$

In this paper, we are interested in the distribution of the statistics numbers of crossings and nestings over the sets $I_{n}(4321), I_{n}(3412)$ and $S_{n}(321,3 \overline{1} 42)$. These sets are all enumerated by the $n$-th Motzkin number (the sequence A001006 in [22]) and they are well studied in the literature (see $[2,6,11]$ and references therein).

Motzkin numbers $M_{n}$ are traditionally defined by the following recurrence relation:

$$
M_{0}=1 \text { and } M_{n}=M_{n-1}+\sum_{k=0}^{n-2} M_{k} M_{n-2-k} \text { for } n \geqslant 1 .
$$


The first values for $M_{n}$ are $1,1,2,4,9,21,51,127,323,835, \ldots$ Using (2), the generating function for Motzkin numbers, i.e. $M(t)=\sum_{n \geqslant 0} M_{n} t^{n}$, satisfies the following equivalent functional equations

$$
M(t)=1+t M(t)+t^{2} M(t)^{2} \text { and } M(t)=\frac{1}{1-t-t^{2} M(t)} .
$$

Solving the first equation of (3) for $M(t)$, we obtain

$$
M(t)=\frac{1-t-\sqrt{1-2 t-3 t^{2}}}{2 t^{2}} .
$$

The second identity of (3) leads to the following continued fraction expansion for $M(t)$

$$
M(t)=\frac{1}{1-t-\frac{t^{2}}{1-t-\frac{t^{2}}{1-t-\frac{t^{2}}{\ddots}}} .}
$$

The term $q$-Motzkin numbers refers to a sequence of polynomials in $q$ whose evaluation at $q=1$ gives the sequence of Motzkin numbers. In other words, this polynomial satisfies the recurrence for the Motzkin numbers and equals to the Motzkin numbers when specializing $q=1$. Some $q$-generalizations of Motzkin numbers are studied by Barcucci et al. [1] and recently by Barnabei and al. [3]. Here, we are interested in two new $q$-Motzkin numbers $M_{n}(q)$ and $\tilde{M}_{n}(q)$. The first one $M_{n}(q)$ is defined by

$$
M_{0}(q)=1, M_{n}(q)=M_{n-1}(q)+\sum_{k=0}^{n-2} q^{k} M_{k}(q) M_{n-2-k}(q) \text { for } n \geqslant 1 .
$$

and the second one $\tilde{M}_{n}(q)$ by

$$
\tilde{M}_{0}(q)=1, \tilde{M}_{n}(q)=\tilde{M}_{n-1}(q)+\sum_{k=0}^{n-2} q^{k+1-(n-1) \delta_{k, n-2}} \tilde{M}_{k}(q) \tilde{M}_{n-2-k}(q) \text { for } n \geqslant 1,
$$

where $\delta$ is the usual Kronecker symbol. With a view to extend the results on $q$-Motzkin numbers introduced in $[1,3]$, we will show how these $q$-Motzkin numbers are the distributions of crossings and nestings over restricted permutations, using continued fractions. For continued fractions, we are inspired by various known applications on enumeration of combinatorial objects (eg. [14, 15, 16, 20, 21]).

The study of the crossings and nestings over permutations was introduced by Médicis and Viennot in [17] and extended in [5, 7, 8, 20, 21]. The study of these statistics over pattern-avoiding permutations was introduced by the second author [18] who exploited a known bijection of Elizalde and Pak [13] to find the equidistributions of the crossings 
over the sets of permutations avoiding the patterns 132, 213, and 321. Later, Rakotomamonjy et al. [19] enumerated the sets of permutations avoiding some pairs of patterns of length 3 according to cr, and they found new combinatorial interpretations of some known sequences in [22]. In this work, we investigate the enumeration of some Motzkin objects, namely the sets $I_{n}(4321), I_{n}(3412)$, and $S_{n}(321,3 \overline{1} 42)$, according to the numbers of crossings and nestings (see Theorem 1). The use of some known bijections between these sets and Motzkin paths allows us to reach our goal.

A Motzkin path of length $n$ is a lattice path starting at $(0,0)$, ending at $(n, 0)$, and never going below the $x$-axis, consisting of up steps $u=(1,1)$, horizontal steps $h=(1,0)$, and down steps $d=(1,-1)$. The set of Motzkin paths of length $n$ will be denoted by $\mathcal{M}_{n}$.

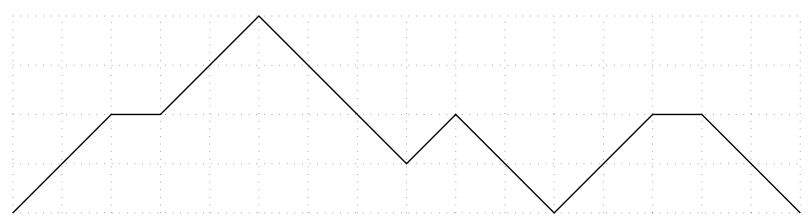

Figure 1: The Motzkin path $P=$ uuhuudddudduuhdd $\in \mathcal{M}_{16}$.

It is well known that the cardinality of $\mathcal{M}_{n}$ is the $n$-th Motzkin number $M_{n}$. The bijections between our combinatorial objects and Motzkin paths are well studied in $[2,6]$. Firstly, Barnabei et al. [2] studied the bijection of Biane [4] restricted to involutions. The bijection of Biane maps an involution into a Motzkin path whose down steps are labelled with an integer that does not exceed their height, while the other steps are unlabelled. Barnabei et al. proved the following characterisations:

- An involution $\tau$ avoids 4321 if and only if the label of any down step in the corresponding Motzkin path is 1 .

- An involution $\tau$ avoids 3412 if and only if the label of any down step in the corresponding Motzkin path equals its height.

Consequently, when restricted to 4321- and 3412-avoiding involutions, we can ignore the labels on Motzkin paths. Secondly, using reduced decomposition of Motzkin paths, Chen et al [6] exhibited a bijection between $S_{n}(321,3 \overline{1} 42)$ and $\mathcal{M}_{n}$ which exchanges the statistics inv and area $-\mathrm{sh}_{u}$. Through these two bijections, we interpret the statistics $\mathrm{cr}$ and nes in terms of statistics on Motzkin paths. Thus, as main results, we find the following combinatorial interpretations of $M_{n}(q)$ and $\tilde{M}_{n}(q)$.

Theorem 1. We have the following identities

$$
\begin{aligned}
& M_{n}(q)=\sum_{\sigma \in I_{n}(4321)} q^{(\mathrm{cr}+\mathrm{nes})(\sigma)}=\sum_{\sigma \in I_{n}(3412)} q^{\operatorname{nes}(\sigma)}, \\
& \tilde{M}_{n}(q)=\sum_{\sigma \in S_{n}(321,3 \overline{1} 42)} q^{\mathrm{cr}(\sigma)} .
\end{aligned}
$$


We organise the rest of this paper in three sections. Section 2 is a preliminary section in which we define some statistics over Motzkin paths and we compute the generating function of their joint distribution. In Section 3 and 4, making use of the result obtained in Section 2 using the bijections of Biane [4] and Chen et al. [6], we compute and express, in terms of continued fractions, the distributions of cr and nes over the sets $I_{n}(4321)$, $I_{n}(3412)$, and $S_{n}(321,3 \overline{1} 42)$. Using the obtained continued fractions, we provide the proof of Theorem 1.

\section{Motzkin paths and statistics}

In this section, we define some statistics over Motzkin paths and we prove a preliminary result that is fundamental for the rest of the paper (see Theorem 5).

Let $P=P_{1} P_{2} \cdots P_{n}$ be a Motzkin path of length $n$. Let us first define the following sets:

$$
\operatorname{Hor}(P):=\left\{i \mid P_{i}=h\right\}, \operatorname{Up}(P):=\left\{i \mid P_{i}=u\right\} \text { and } \operatorname{Down}(P):=\left\{i \mid P_{i}=d\right\} .
$$

The height of each step $s$ in $P$ is the $y$-coordinate of the starting point of $s$. Therefore, the height of the $i$-th step of $P$ is equal to

$$
h_{i}(P)= \begin{cases}|P(1, i)|_{u}-|P(1, i)|_{d}-1 ; & \text { if } P_{i}=u \\ |P(1, i)|_{u}-|P(1, i)|_{d} ; & \text { if } P_{i}=h, \\ |P(1, i)|_{u}-|P(1, i)|_{d}+1 ; & \text { if } P_{i}=d\end{cases}
$$

where $P(1, i):=P_{1} P_{2} \cdots P_{i}$ is the initial sub-path of length $i$ of $P$ and, for any word $w$, $|w|_{a}$ is the number of occurrences of the letter $a$ in the word $w$. Here are some statistics over $\mathcal{M}_{n}$ :

- $\operatorname{area}(P)$ the area between the path $P$ and the $x$-axis,

- $\operatorname{up}(P)$ (resp. hor $(P)$, down $(P))$ the number of up (resp. horizontal, down) steps of $P$,

- $\operatorname{sh}_{u}(P)\left(\operatorname{resp} . \operatorname{sh}_{h}(P), \operatorname{sh}_{d}(P)\right)$ the sum of heights of all up (resp. horizontal, down) steps of $P$, i.e.

$$
\operatorname{sh}_{u}(P):=\sum_{i \in U p(P)} h_{i}(P), \operatorname{sh}_{h}(P):=\sum_{i \in \operatorname{Hor}(P)} h_{i}(P), \text { and } \operatorname{sh}_{d}(P):=\sum_{i \in \operatorname{Down}(P)} h_{i}(P) .
$$

For example, the Motzkin path in Fig. 1 has the following properties: $\operatorname{hor}(P)=2$, $\operatorname{up}(P)=\operatorname{down}(P)=7, \operatorname{sh}_{u}(P)=8, \operatorname{sh}_{h}(P)=4, \operatorname{sh}_{d}(P)=15$ and area $(P)=27$. We will show how these statistics are related.

Proposition 2. For any Motzkin path $P$, we have

$$
\operatorname{area}(P)=2 \operatorname{sh}_{d}(P)+\operatorname{sh}_{h}(P)-\operatorname{down}(P) .
$$


Proof. The proof is simply based on the following properties.

- If the $i$-th step of $P$ is a down step of height $h$, the area of the polygon $(i-1, h)-$ $(i, h-1)-(i+1-h, 0)-(i-1-h, 0)$ is $2 h-1$.

- If the $i$-th step of $P$ is a horizontal step of height $h$, the area of the polygon $(i-$ $1, h)-(i, h)-(i+1-h, 0)-(i-h, 0)$ is $h$.

The area of the path $P$ is equal to the sum of the areas of such polygons corresponding to down and horizontal steps (see Figure 2).

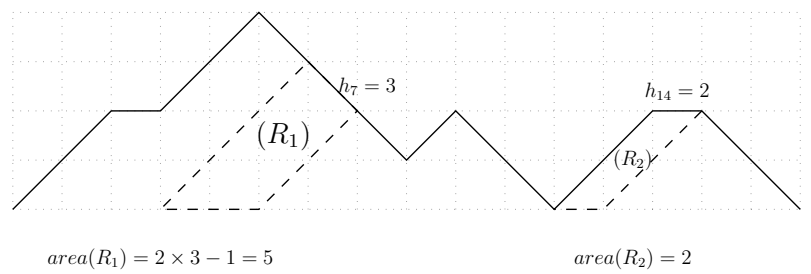

Figure 2: Decomposition of the area of a Motzkin path.

By the same way, when we decompose the area statistic according to up and horizontal steps, we obtain the following proposition.

Proposition 3. For any Motzkin path P, we have

$$
\operatorname{area}(P)=2 \operatorname{sh}_{u}(P)+\operatorname{sh}_{h}(P)+u p(P) .
$$

Corollary 4. For any Motzkin path $P$, we have $\operatorname{sh}_{u}(P)=\operatorname{sh}_{d}(P)-\operatorname{down}(P)$.

Proof. Using both propositions 2 and 3 with the obvious fact that $\operatorname{up}(P)=\operatorname{down}(P)$ for any Motzkin path $P$, we obtain the corollary.

We now consider the following polynomial

$$
I_{n}(a, b, c, d)=\sum_{P \in \mathcal{M}_{n}} a^{\operatorname{hor}(P)} b^{\operatorname{up}(P)} c^{\mathrm{sh}_{u}(P)} d^{\mathrm{sh}_{h}(P)},
$$

and we denote by $I(a, b, c, d ; t)=\sum_{n \geqslant 0} I_{n}(a, b, c, d) t^{n}$ the generating function of $I_{n}(a, b, c, d)$. Before closing this section, we will prove a fundamental identity which allows us to get easily the main results of this paper.

Theorem 5. The continued fraction expansion of $I(a, b, c, d ; t)$ is

$$
\frac{1}{1-a t-\frac{b t^{2}}{1-a d t-\frac{b c t^{2}}{1-a d^{2} t-\frac{b c^{2} t^{2}}{\ddots}}} .}
$$


Proof. The proof is based on the usual decomposition of Motzkin paths. Let $P \in \mathcal{M}_{n}$. Using the first return decomposition, we obtain the following relations:

- if $P=h P^{\prime}$ for some $P^{\prime} \in \mathcal{M}_{n-1}$, then we have

$$
\text { (hor, up, } \left.\operatorname{sh}_{u}, \operatorname{sh}_{h}\right) P=\left(1+\text { hor, up, } \operatorname{sh}_{u}, \operatorname{sh}_{h}\right) P^{\prime} \text {. }
$$

- If $P=u P_{1} d P_{2}$ for some $k \geqslant 2$ such that $P_{1} \in \mathcal{M}_{k-2}$ and $P_{2} \in \mathcal{M}_{n-k}$, then we have

$$
\text { (hor, up, } \left.\operatorname{sh}_{u}, \operatorname{sh}_{h}\right) P=\left(\text { hor, } 1+\text { up, } \operatorname{sh}_{u}+\text { up, } \operatorname{sh}_{h}+\text { hor }\right) P_{1}+\left(\text { hor, up, } \operatorname{sh}_{u}, \operatorname{sh}_{h}\right) P_{2} \text {. }
$$

These two points lead to the following recurrence for the polynomial $I_{n}(a, b, c, d)$ :

$$
I_{n}(a, b, c, d)=a I_{n-1}(a, b, c, d)+b \sum_{k=2}^{n} I_{k-2}(a d, b c, c, d) I_{n-k}(a, b, c, d),
$$

with $I_{0}(a, b, c, d)=1$. Thus, when we compute $I(a, b, c, d ; t)$, we obtain from (8) the following relation

$$
I(a, b, c, d ; t)=\frac{1}{1-a t-b t^{2} I(a d, b c, c, d ; t)} .
$$

Developing $I(a d, b c, c, d ; t)$ in its turn, we obtain the desired continued fraction expansion for $I(a, b, c, d ; t)$.

\section{Crossings and nestings over $I_{n}(4321)$ and $I_{n}(3412)$}

In this section, we will establish the proof of the first part of Theorem 1 concerning the recursion for the polynomial distribution of crossings and nestings over the sets $I_{n}(4321)$ and $I_{n}(3412)$. For that, we first recall the bijection of Biane [4] restricted to the set of involutions that was studied by Barnabei et al. [2]. The bijection maps an involution into a Motzkin path whose down steps are labelled with an integer that does not exceed its height, while the other steps are unlabelled. Barnabei et al. [2] proved the following characterisations:

- An involution $\sigma$ avoids 4321 if and only if the label of any down step in the corresponding Motzkin path is 1 .

- An involution $\sigma$ avoids 3412 if and only if the label of any down step in the corresponding Motzkin path equals its height.

Consequently, when restricted to 4321- and 3412-avoiding involutions, we can ignore the labels on Motzkin paths. Let us denote by $\Phi_{1}$ (resp. $\Phi_{2}$ ) the bijection from $\mathcal{M}_{n}$ to $I_{n}(4321)$ (resp. $\left.I_{n}(3412)\right)$. In this section, we manipulate the bijections $\Phi_{1}$ and $\Phi_{2}$, and we will show how these bijections interchange the statistics (fp, exc, cr, nes) and (hor, up, $a \cdot \mathrm{sh}_{u}, b \cdot \mathrm{sh}_{h}$ ), where $a$ and $b$ are two arbitrary integers. 
Since the construction of the image of an involution under the maps $\Phi_{1}$ and $\Phi_{2}$ is the same and obvious (see [2]), we just focus on how to get the corresponding involutions from Motzkin paths. Let $P \in \mathcal{M}_{n}$. We will construct $\sigma_{1}=\Phi_{1}(P)$ by the following procedure (see Figure 3 for graphical illustration).

- From left to right, number the steps of $P$ from 1 up to $n$.

- Read the steps of $P$ from left to right and match the $k$-th up step with the $k$-th down step, $k=1,2, \ldots$.

- Then, we have

(a) $(i)$ is a 1-cycle of $\sigma_{1}$, i.e. $\sigma_{1}(i)=i$, if and only if the step numbered $i$ is horizontal.

(b) $(i, j)$ is a 2-cycle of $\sigma_{1}$, i.e. $\sigma_{1}(i)=j$ and $\sigma_{1}(j)=i$, if and only if the $i$-th and the $j$-th step are matched.

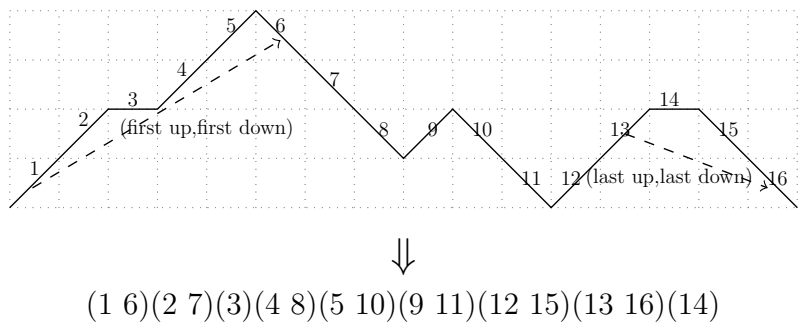

Figure 3: The corresponding 4321-avoiding involution from a Motzkin path.

Before giving the formulation of $\Phi_{2}$, we first recall what a tunnel is. The notion of tunnels on Dyck paths (Dyck paths are Motzkin paths having no horizontal step) is introduced by Elizalde et al. [12, 13] and Barnabey et al. [3] extend the notion in terms of Motzkin paths. A tunnel of a Motzkin path $P$ is a horizontal segment between two distinct lattice points of $P$ that intersects $P$ only at these two points and lies always below $P$. Each tunnel $t$ starts from the starting point of an up step $u$ to the ending point of a down step $d$. We say that the tunnel $t$ matches $u$ and $d$. For example, the Motzkin path in Figure 4 has 7 tunnels drawn in right dashed arrows. In particular, the tunnel $t_{3}$ matches the up step numbered 4 and the down step numbered 7. For any Motzkin path $P$, we can construct $\sigma_{2}=\Phi_{2}(P)$ by the following procedure (see Figure 4):

- From left to right, number the steps of $P$ from 1 up to $n$.

- Then, we have

(a) $(i)$ is a 1-cycle of $\sigma_{2}$ if and only if the step numbered $i$ is horizontal.

(b) $(i, j)$ is a 2-cycle of $\sigma_{2}$ if and only if the up step numbered $i$ is matched with the down step numbered $j$ by a tunnel. 


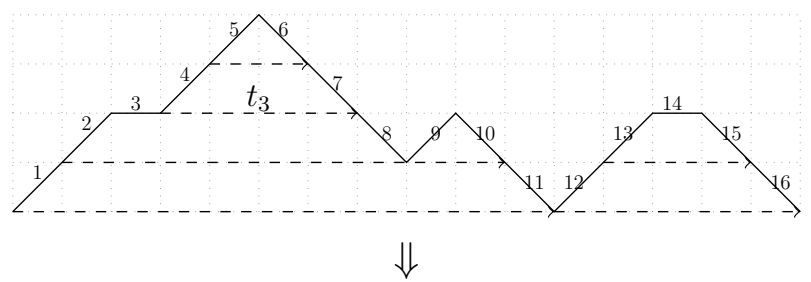

$(1$ 11)(2 8)(3)(4 7)(5 6)(9 10)(12 16)(13 15)(14)

Figure 4: The corresponding 3412-avoiding involution from a Motzkin path.

By these definitions, it is not difficult to show that the bijections $\Phi_{1}$ and $\Phi_{2}$ are well defined.

Proposition 6. Let $P$ be a Motzkin path. Assume that $\sigma_{1}=\Phi_{1}(P)$ and $\sigma_{2}=\Phi_{2}(P)$. We have the following properties

(i) $(\mathrm{cr}$, nes $)\left(\sigma_{1}\right)=\left(2 \mathrm{sh}_{u}, \operatorname{sh}_{h}\right)(P)$.

(ii) $\operatorname{nes}\left(\sigma_{2}\right)=\left(2 \operatorname{sh}_{u}+\operatorname{sh}_{h}\right)(P)$.

Proof. Let $\sigma$ be an involution. By definition, we have

$$
\begin{aligned}
\operatorname{nes}(\sigma) & =2 \sum_{\sigma(i)>i}|\{j<i \mid \sigma(j)>\sigma(i)\}|+\sum_{\sigma(i)=i}|\{j>i \mid \sigma(j)<i\}|, \\
\text { and } \operatorname{cr}(\sigma) & =2 \sum_{\sigma(i)>i}|\{i<j<\sigma(i) \mid \sigma(j)>\sigma(i)\}| .
\end{aligned}
$$

Notice first that the following properties are obvious:

(a) if $\sigma$ is 4321-avoiding, then we have $|\{j<i \mid \sigma(j)>\sigma(i)\}|=0$ for all $i$ such that $\sigma(i)>i$.

(b) if $\sigma$ is 3412-avoiding, then we have $\operatorname{cr}(\sigma)=0$.

Let us suppose that $\sigma_{1}=\Phi_{1}(P)$ for some Motzkin path $P$. According to (a), we have

$$
\begin{aligned}
\operatorname{nes}\left(\sigma_{1}\right) & =\sum_{\sigma_{1}(i)=i}\left|\left\{j>i \mid \sigma_{1}(j)>i\right\}\right| \\
& =\sum_{\sigma_{1}(i)=i}\left|\left\{k<i \mid \sigma_{1}(k)>i\right\}\right| \\
& =\sum_{i \in \operatorname{Hor}(P)}\left(|P(1, i)|_{u}-|P(1, i)|_{d}\right), \\
& =\operatorname{sh}_{h}(P) .
\end{aligned}
$$


Moreover, according to the given definition of $\Phi_{1}$, we have $\mid\left\{i<j<\sigma_{1}(i) \mid \sigma_{1}(j)>\right.$ $\left.\sigma_{1}(i)\right\}\left.|=| P\left(1, \sigma_{1}(i)\right)\right|_{u}-\left|P\left(1, \sigma_{1}(i)\right)\right|_{d}=h_{\sigma_{1}(i)}(P)-1$ (with $\sigma_{1}(i)$ as down step of $\left.\sigma_{1}\right)$. Consequently, we get

$$
\begin{aligned}
\operatorname{cr}\left(\sigma_{1}\right) & =2 \sum_{\sigma_{1}(i)>i}\left|\left\{i<j<\sigma_{1}(i) \mid \sigma_{1}(j)>\sigma_{1}(i)\right\}\right| \\
& =2 \sum_{\sigma_{1}(i)>i}\left(h_{\sigma_{1}(i)}(P)-1\right) \\
& =2 \sum_{l \in \text { Down }(P)}\left(h_{l}(P)-1\right) \\
& =2\left(\operatorname{sh}_{d}(P)-\operatorname{down}(P)\right) \\
& =2 \operatorname{sh}_{u}(P) \quad(\text { see Corollary } 4) .
\end{aligned}
$$

This ends the proof of (i). To prove (ii), we now suppose that $\sigma_{2}=\Phi_{2}(P)$ for some Motzkin path $P$. For any excedance $i$ of $\sigma_{2}$, we have $\left.\left|\left\{j<i \mid \sigma_{2}(j)>\sigma_{2}(i)\right\}\right|=\mid P(1, i)\right)\left.\right|_{u}-$ $|P(1, i)|_{d}=h_{i}(P)$ and $i \in U p(P)$.

$$
\begin{aligned}
\operatorname{nes}\left(\sigma_{2}\right) & =2 \sum_{\sigma_{2}(i)>i}\left|\left\{j<i \mid \sigma_{2}(j)>\sigma_{2}(i)\right\}\right|+\sum_{\sigma(i)=i}\left|\left\{j>i \mid \sigma_{2}(j)>i\right\}\right|, \\
& =2 \sum_{i \in U p(P)} h_{i}(P)+\sum_{i \in \operatorname{Hor}(P)} h_{i}(P), \\
& =2 \operatorname{sh}_{u}(P)+\operatorname{sh}_{h}(P) .
\end{aligned}
$$

This also ends the proof of (ii). Thus, Proposition 6 follows.

We now have all necessary tools to prove the following results concerning the joint distribution of the statistics fp, exc, cr and nes over our sets of 4321- and 3412-avoiding involutions.

Theorem 7. We have the following identities

$$
\sum_{\sigma \in I(4321)} x^{\mathrm{fp}(\sigma)} y^{\operatorname{exc}(\sigma)} p^{\mathrm{cr}(\sigma)} q^{\mathrm{nes}(\sigma)} t^{|\sigma|}=\frac{1}{1-x t-\frac{y t^{2}}{1-x q t-\frac{y p^{2} t^{2}}{1-x q^{2} t-\frac{y p^{4} t^{2}}{\ddots}}}},
$$

and

$$
\sum_{\sigma \in I(3412)} x^{\mathrm{fp}(\sigma)} y^{\operatorname{exc}(\sigma)} q^{\mathrm{nes}(\sigma)} t^{|\sigma|}=\frac{1}{1-x t-\frac{y t^{2}}{1-x q t-\frac{y q^{2} t^{2}}{1-x q^{2} t-\frac{y q^{4} t^{2}}{\cdot}}}} .
$$


Proof. It is obvious to see from their definitions that the bijections $\Phi_{1}$ and $\Phi_{2}$ interchange the statistics (fp, exc) and (hor, up) (or (hor, down)). So, using Proposition 6, we get

- (hor, up, $\left.2 \mathrm{sh}_{u}, \mathrm{sh}_{h}\right) \stackrel{\Phi_{1}}{\longrightarrow}$ (fp, exc, cr, nes)

- and (hor, up, $\left.2 \mathrm{sh}_{u}+\mathrm{sh}_{h}\right) \stackrel{\Phi_{2}}{\longrightarrow}$ (fp, exc, nes)

Consequently, we have

$$
\begin{aligned}
\sum_{\sigma \in I_{n}(4321)} x^{\mathrm{fp}(\sigma)} y^{\operatorname{exc}(\sigma)} p^{\operatorname{cr}(\sigma)} q^{\operatorname{nes}(\sigma)} & =I_{n}\left(x, y, p^{2}, q\right), \\
\text { and } \sum_{\sigma \in I_{n}(3412)} x^{\mathrm{fp}(\sigma)} y^{\operatorname{exc}(\sigma)} q^{\operatorname{nes}(\sigma)} & =I_{n}\left(x, y, q^{2}, q\right) .
\end{aligned}
$$

So, we obtain easily identity (9) (resp. (10)) from (7), by setting $a=x, b=y, c=p^{2}$ (resp. $c=q^{2}$ ) and $d=q$. This ends the proof of Theorem 7 .

Now, to close this section, we will prove the first identities of the main result presented in the introduction (see. Theorem 1).

Theorem 8. We have $\sum_{\sigma \in I_{n}(4321)} q^{(\mathrm{cr}+\mathrm{nes})(\sigma)}=\sum_{\sigma \in I_{n}(4312)} q^{\operatorname{cr}(\sigma)}=M_{n}(q)$.

Proof. Let us denote by

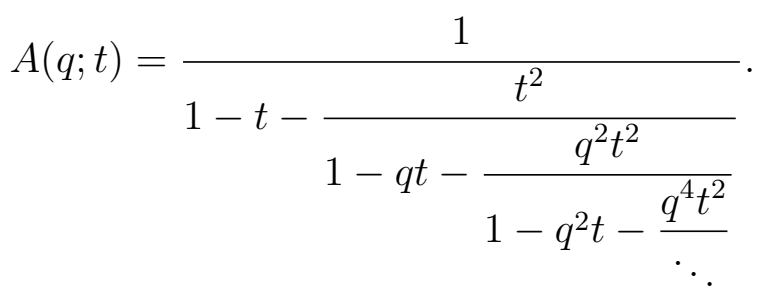

Simple manipulation of the continued fraction expansion of $A(q ; t)$ leads to the following functional equation

$$
A(q ; t)=\frac{1}{1-t-t^{2} A(q ; q t)} .
$$

This is also equivalent to the following one

$$
A(q ; t)=1+t A(q ; t)+t^{2} A(q ; t) A(q ; q t) .
$$

When we extract the coefficient $A_{n}(q)$ of $t^{n}$ on both sides of this equation, we obtain

$$
A_{0}(q)=1, A_{n}(q)=A_{n-1}(q)+\sum_{k=0}^{n-2} q^{k} A_{k}(q) A_{n-2-k}(q) \text { for } n \geqslant 1 .
$$

Since the polynomials $A_{n}(q)$ and $M_{n}(q)$ have the same recurrence (see recursion $(2)$ ) and the same initial values, so they are the same. Furthermore, from (9) and (10), we obtain

$$
\sum_{\sigma \in I(4321)} q^{(\mathrm{cr}+\mathrm{nes})(\sigma)} t^{|\sigma|}=\sum_{\sigma \in I(3412)} q^{\mathrm{cr}(\sigma)} t^{|\sigma|}=A(q ; t)
$$

Thus, we get Theorem 8 . 


\section{Crossings over $S_{n}(321,3 \overline{1} 42)$}

The main goal of this section is to prove the second part of Theorem 1 concerning the distribution of crossings over $S_{n}(321,3 \overline{1} 42)$. For that, we will use the bijection of Chen et al. [6] based on reduced decomposition of permutations and strip decomposition of Motzkin paths.

A transposition $s_{i}=(i i+1)$ (i.e. 2-cycle) is a map from $S_{n}$ to itself which interchanges the numbers in the $i$ th and $(i+1)$ th position in a permutation. For example, $s_{3}(623514)=$ 625314. Every permutation $\sigma$ in $S_{n}$ can be represented as a sequence of transpositions $s_{i_{1}} s_{i_{2}} \cdots s_{i_{k}}$ which, when applied from right to left to the identity permutation $123 \cdots n$, results in $\sigma$. Such representation is not necessarily unique. For example, 321 can be written as both $s_{1} s_{2} s_{1}$ and $s_{2} s_{1} s_{2}$. In fact, the product of simple transpositions satisfies the Braid relations:

$$
s_{i+1} s_{i} s_{i+1}=s_{i} s_{i+1} s_{i} \text { and } s_{i} s_{j}=s_{j} s_{i}, \text { where }|i-j| \neq 1 .
$$

Let $\sigma \in S_{n}$. We now determine an unique representation corresponding to $\sigma$, called the canonical reduced decomposition of $\sigma$, by a specific procedure. For that, we denote by $\mathcal{R}(\sigma)$ the set of the pairs resulting from the following procedure and initiate it to the empty set.

1. If $\sigma$ is the identity, then the corresponding canonical reduced decomposition of $\sigma$ is the identity. Otherwise, we let $\sigma^{(0)}:=\sigma$ and we go to the next step.

2. Locate the pair $\left(n_{1}, i_{1}\right)$, where $n_{1}$ is the greatest excedance value of $\sigma^{(0)}$ and $i_{1}$ its position (i.e. $i_{1}<n_{1}=\sigma^{(0)}\left(i_{1}\right)$ since $i_{1}$ is an excedance of $\left.\sigma^{(0)}\right)$. Move $n_{1}$ from $i_{1}$ to position $n_{1}$ by applying from right to left the sequence $s_{n_{1}-1} s_{n_{1}-2} \cdots s_{i_{1}+1} s_{i_{1}}$ and leave the relative order of the other numbers unchanged. Let $\sigma^{(1)}$ be the resulting permutation. Then, add the pair $\left(n_{1}-1, i_{1}\right)$ to $\mathcal{R}(\sigma)$ and pass to the next step.

3. Return to the first step and apply the procedure to $\sigma^{(1)}$.

By this manner, if we have $\mathcal{R}(\sigma)=\left\{\left(h_{1}, t_{1}\right),\left(h_{2}, t_{2}\right), \ldots,\left(h_{k}, t_{k}\right)\right\}$, i.e. $\left(h_{k+1-j}, t_{k+1-j}\right)=$ $\left(n_{j}-1, i_{j}\right)$ for any $1 \leqslant j \leqslant k$, then the canonical reduced decomposition of $\sigma$ is $\sigma_{1} \sigma_{2} \cdots \sigma_{k}$, where $\sigma_{j}=s_{h_{j}} s_{h_{j}-1} \cdots s_{t_{j}}$ for any $1 \leqslant j \leqslant k$. It is known that such decomposition is unique. We have to notice that the above algorithm is none other than that of Chen et al.[6]. We just modified it to emphasize the notion of excedance. In Chen et al. [6], the set $\mathcal{R}(\sigma)$ is known as the set of pairs (head,tail) and they showed the following characterisation.

Theorem 9. [6, Thm. 2.3] Let $\sigma \in S_{n}$ and $\mathcal{R}(\sigma)=\left\{\left(h_{1}, t_{1}\right),\left(h_{2}, t_{2}\right), \ldots,\left(h_{k}, t_{k}\right)\right\}$. We have $\sigma \in S_{n}(321,3 \overline{1} 42)$ if and only if $t_{j-1}+2 \leqslant t_{j}$ for $1<j \leqslant k$.

Here are some examples,

- the canonical reduced decomposition of the identity $i d=12 \cdots n$ is the identity since $\mathcal{R}(i d)=\emptyset$; 
- the canonical reduced decomposition of $\pi=n n-1 \cdots 21$ is $\pi$ is $\pi=s_{1} / s_{2} s_{1} /$ $s_{3} s_{2} s_{1} / \cdots / s_{n-1} s_{n-2} \cdots s_{1}$ since $\mathcal{R}(\pi)=\{(1,1),(2,1), \ldots,(n-1,1)\}$;

- if $\sigma=61723841051191512161314$, we have

$$
\mathcal{R}(\sigma)=\{(5,1),(6,3),(7,6),(9,8),(10,10),(14,12),(15,14)\} .
$$

Thus, the canonical reduced decomposition of $\sigma$ is $\sigma_{1} \sigma_{2} \cdots \sigma_{7}$, with $\sigma_{1}=s_{5} s_{4} s_{3} s_{2} s_{1}$, $\sigma_{2}=s_{6} s_{5} s_{4} s_{3}, \sigma_{3}=s_{7} s_{6}, \sigma_{4}=s_{9} s_{8}, \sigma_{5}=s_{10}, \sigma_{6}=s_{14} s_{13} s_{12}$, and $\sigma_{7}=s_{15} s_{14}$.

The following theorem is an extension of [6, Thm. 2.4].

Theorem 10. [6] For any $\sigma \in S_{n}(321,3 \overline{1} 42)$, if $\mathcal{R}(\sigma)=\left\{\left(h_{r}, t_{r}\right)\right\}_{1 \leqslant r \leqslant k}$, then we have $\operatorname{Des}(\sigma)=\operatorname{Exc}(\sigma)=\left\{t_{1}, t_{2}, \ldots, t_{k}\right\}$.

Proof. Let $\sigma \in S_{n}(321,3 \overline{1} 42)$. Since $\sigma$ is 321-avoiding, then nes $(\sigma)=0$ (see. [18, Lem. 5.1]). Consequently, if $i_{1}<i_{2}<\cdots<i_{k}$ and $j_{1}<j_{2}<\cdots<j_{n-k}$ are respectively excedances and non-excedances of $\sigma$, then we have $\sigma\left(i_{1}\right)<\sigma\left(i_{2}\right)<\cdots<\sigma\left(i_{k}\right)$ and $\sigma\left(j_{1}\right)<\sigma\left(j_{2}\right)<\cdots<\sigma\left(j_{n-k}\right)$. It is clear that, when we apply the above procedure, we get $\mathcal{R}(\sigma)=\left\{\left(\sigma\left(i_{1}\right)-1, i_{1}\right),\left(\sigma\left(i_{2}\right)-1, i_{2}\right), \ldots,\left(\sigma\left(i_{k}\right)-1, i_{k}\right)\right\}$. So, we have $\operatorname{Exc}(\sigma)=$ $\left\{i_{1}, i_{2}, \ldots, i_{k}\right\}$. For any $1 \leqslant j \leqslant k$, since $i_{j}+2<i_{j+1}$ (see Theorem 9 ), then $i_{j}+1$ is a non-excedance of $\sigma$ and consequently we have $\sigma\left(i_{j}\right)>\sigma\left(i_{j}+1\right)$. Thus, excedances of $\sigma$ are all descents of $\sigma$. Furthermore, for some $x$, a non-excedance $j_{x}$ of $\sigma$ can not be descent of $\sigma$ because $j_{x}$ is followed by, either an excedance and we have $\sigma\left(j_{x}\right)<j_{x}+1<\sigma\left(j_{x}+1\right)$, or a non-excedance and we have $\sigma\left(j_{x}\right)<\sigma\left(j_{x}+1\right)<j_{x}+1$. Thus, we get $\operatorname{Des}(\sigma)=\operatorname{Exc}(\sigma)$.

Now, we recall the $(\mathrm{x}+\mathrm{y})$-labelling and the strip decomposition of a Motzkin path. We call the $(x+y)$-labelling of a Motzkin path $P$ the action of labelling some cells (squares and triangulars containing a down-step) between $P$ and the $x$-axis by the sum of the coordinates of their left bottom coin. We now define the strip decomposition of a Motzkin path. Suppose $P=P_{n, k}$ is a Motzkin path of length $n$ that contains $k$ up steps. If $k=0$, then the strip decomposition of $P_{n, 0}$ is simply the empty set. For any $P_{n, k} \in \mathcal{M}_{n}$, let $A \rightarrow B$ be the last up step and $E \rightarrow F$ the last down step on $P_{n, k}$. Then we define the strip of $P_{n, k}$ as the path from $B$ to $F$ along the path $P_{n, k}$. Now we move the points from $B$ to $E$ one layer lower, namely, subtract the $y$-coordinate by 1 , and denote the adjusted points by $B_{0}, \ldots, E_{0}$. We form a new Motzkin path by using the path $P_{n, k}$ up to the point $A$, then joining the point $A$ to $B_{0}$ and following the adjusted segment until we reach the point $E_{0}$, then continuing with the points on the $x$-axis to reach the destination $(n, 0)$. Denote this Motzkin path by $P_{n, k-1}$, which may end with some horizontal steps. From the strip of $P_{n, k}$, we may define the value $h_{k}$ as the label of the cell containing the step $E \rightarrow F$. Clearly, we have $h_{k} \leqslant n-1$. The value $t_{k}$ is defined as the label of the cell containing the step starting from the point $B$.

Iterating the above procedure, we obtain the set $\mathcal{S}(P):=\left\{\left(h_{i}, t_{i}\right)\right\}_{1 \leqslant i \leqslant k}$, called the strip decomposition of $P$, satisfying

$$
h_{j}<h_{j+1} \text { and } t_{j}+2<t_{j+1} \text { for any } 1 \leqslant i<k .
$$


The strip decomposition of the Motzkin path $P=$ uuhduudddudduuhdd drawn in Figure 5 is $\mathcal{S}(P)=\{(5,1),(6,3),(7,6),(9,8),(10,10),(14,12),(15,14)\}$. It is known that every Motzkin path $P$ can be determined from the set $\mathcal{S}(P)$ satisfying (11) by reversing the above procedure ( see [6, Fig. 2]).

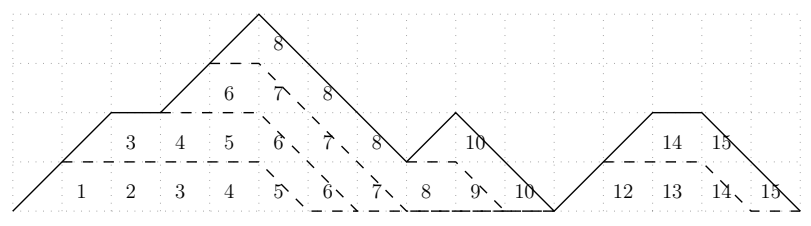

Figure 5: The $(x+y)$-labelling and the strip decomposition of a Motzkin path.

According to Theorem 9, each strip decomposition of a Motzkin path $P$, i.e. $\mathcal{S}(P):=$ $\left\{\left(h_{i}, t_{i}\right)\right\}_{1 \leqslant i \leqslant k}$, satisfying 11 is associated with a permutation $\sigma \in S_{n}(321,3 \overline{1} 42)$ throughout its reduced decomposition deduced from $\mathcal{R}(\sigma)=\left\{\left(h_{i}, t_{i}\right)\right\}_{1 \leqslant i \leqslant k}$, i.e. $\sigma_{1} \sigma_{2} \cdots \sigma_{k}$, where $\sigma_{j}=s_{h_{j}} s_{h_{j}-1} \cdots s_{t_{j}}$. In other words, the bijection $\Phi_{3}$ of Chen et al. from $\mathcal{M}_{n}$ to $S_{n}(321,3 \overline{1} 42)$ is defined as follows: for any $P \in \mathcal{M}_{n}$

$$
\sigma=\Phi_{3}(P) \text { if and only if } \mathcal{R}(\sigma)=\mathcal{S}(P) .
$$

Proposition 11. Let $P \in \mathcal{M}_{n}$ and $\sigma=\Phi_{3}(P)$. We have

$$
(\operatorname{exc}, \mathrm{cr})(\sigma)=\left(\mathrm{up}, \operatorname{sh}_{u}+\operatorname{sh}_{h}\right)(P) \text {. }
$$

Proof. Since the strip decomposition of $P$ is associated with up steps, we have $\operatorname{up}(P)=$ $|\mathcal{S}(P)|=|\mathcal{R}(\sigma)|$. Moreover, using Theorem 10, we obtain $|\mathcal{R}(\sigma)|=\operatorname{exc}(\sigma)$. So, we get $\operatorname{up}(P)=\operatorname{exc}(\sigma)$. Chen et al. proved that we have $\operatorname{inv}(\sigma)=\left(\operatorname{area}-\operatorname{sh}_{u}\right)(P)($ see $[6$, Thm. $3.2])$. Finally, we get

$$
\begin{aligned}
\operatorname{cr}(\sigma) & =\operatorname{inv}(\sigma)-\operatorname{exc}(\sigma) \text { using }(1) \text { with nes }(\sigma)=0 \\
& =\operatorname{area}(P)-\operatorname{sh}_{u}(P)-\operatorname{up}(P) \\
& =\operatorname{sh}_{u}(P)+\operatorname{sh}_{h}(P) \text { using }(6) \text { of Proposition } 3 .
\end{aligned}
$$

This ends the proof of our proposition.

Theorem 12. We have

$$
\sum_{\sigma \in S(321,3 \overline{1} 42)} y^{\operatorname{exc}(\sigma)} q^{\mathrm{cr}(\sigma)} t^{|\sigma|}=\frac{1}{1-t-\frac{y t^{2}}{1-q t-\frac{y q t^{2}}{1-q^{2} t-\frac{y q^{2} t^{2}}{\ddots}}} .}
$$

Proof. By the preceding proposition, we can get $\sum_{\sigma \in S_{n}(321,3 \overline{1} 42)} y^{\operatorname{exc}(\sigma)} q^{\operatorname{cr}(\sigma)}=I_{n}(1, y, q, q)$. By setting $a=1, b=y$ and $c=d=q$ in (7), we obtain the theorem. 
We now establish the proof of the second part of our main result concerning the recursion for the polynomials $\sum_{\sigma} q^{\operatorname{cr}(\sigma)}$, where the sum runs over all $\sigma \in S_{n}(321,3 \overline{1} 42)$. For that, we recall the Stieltjes tableau $\left(h_{n, i}\right)$ of two sequences $\left(\alpha_{i}\right)$ and $\left(\beta_{i}\right)$ considered by Dumont [9] in his master course on the $J$-continued fractions and Motzkin paths. He defined the tableau $\left(h_{n, i}\right)$ through the following recurrence elsewhere

$$
\left\{\begin{array}{l}
h_{0,0}=1, \text { and } h_{n, i}=0 \text { if } i<0 \text { or } i>n \\
h_{n, i}=\beta_{i} h_{n-1, i-1}+\alpha_{i+1} h_{n-1, i}+h_{n-1, i+1}, \text { elsewhere. }
\end{array}\right.
$$

Using recursion (13), Dumont showed the following theorem. To prove it, we can we can inquire into the paper of Dumont and Randrianarivony [10].

Theorem 13. [9] We have

$$
\frac{1}{1-\alpha_{1} t-\frac{\beta_{1} t^{2}}{1-\alpha_{2} t-\frac{\beta_{2} t^{2}}{1-\alpha_{3} t-\frac{\beta_{3} t^{2}}{\cdot}}}}=h_{0,0}+h_{1,0} t+h_{2,0} t^{2}+\cdots+h_{n, 0} t^{n}+\cdots .
$$

Here, we are interested in the particular Stieltjes tableau $\left(H_{n, i}\right)$ obtained from $\left(\alpha_{i}\right)$ and $\left(\beta_{i}\right)$ with $\alpha_{i}=\beta_{i}=q^{i-1}$ for any integer $i \geqslant 0$, i.e.

$$
\left\{\begin{array}{l}
H_{0,0}=1, \text { and } H_{n, i}=0 \text { if } i<0 \text { or } i>n \\
H_{n, i}=q^{i-1} H_{n-1, i-1}+q^{i} H_{n-1, i}+H_{n-1, i+1}, \text { elsewhere. }
\end{array}\right.
$$

As direct corollary of Theorem 13, we have

$$
\frac{1}{1-t-\frac{t^{2}}{1-q t-\frac{q t^{2}}{1-q^{2} t-\frac{q^{2} t^{2}}{\cdot}}}}=H_{0,0}+H_{1,0} t+H_{2,0} t^{2}+\cdots+H_{n, 0} t^{n}+\cdots
$$

We will prove the following unexpected recursion satisfied by the sequence $\left(H_{n, i}\right)$.

Theorem 14. For any integers $n \geqslant 1$ and $1 \leqslant i \leqslant n$, we have

$$
H_{n, i}=q^{i-1}\left(H_{n-1, i-1}+\sum_{k=i-1}^{n-2} q^{1+k} H_{k, i-1} H_{n-1-k, 0}\right) .
$$

Proof. To prove (17), we will proceed by induction on $n$. For that, we can easily verify that (17) holds for $n=1,2,3$. Suppose that it holds until some integer $n$. To prove that 


\begin{tabular}{c|lllll}
$n / k$ & 0 & 1 & 2 & 3 & 4 \\
\hline 0 & 1 & & & & \\
1 & 1 & 1 & & & \\
2 & 2 & $1+q$ & $q$ & $q^{3}$ & \\
3 & $3+q$ & $2+2 q+q^{2}$ & $q+q^{2}+q^{3}$ & $q^{3}+q^{4}+q^{5}+q^{6}$ & $q^{6}$
\end{tabular}

Table 1: Values of the $q$-tableau $\left(H_{n, i}\right)$ for $0 \leqslant n, i \leqslant 4$.

it still holds for $n+1$, we treat separately the cases $i=1$ and $i \geqslant 2$. By definition, we have

$$
H_{n+1,1}=H_{n, 0}+q H_{n, 1}+H_{n, 2} .
$$

For $H_{n, 2}$, we use (17) and the relationship $H_{k, 1}=H_{k+1,0}-H_{k, 0}$ for $k \geqslant 1$ to obtain

$$
\begin{aligned}
H_{n, 2} & =q\left(H_{n-1,1}+\sum_{k=1}^{n-2} q^{1+k} H_{k, 1} H_{n-1-k, 0}\right) \\
& =q\left(H_{n, 0}-H_{n-1,0}+\sum_{k=1}^{n-2} q^{1+k}\left(H_{k+1,0}-H_{k, 0}\right) H_{n-1-k, 0}\right) \\
& =q\left(H_{n, 0}+\sum_{k=2}^{n-1} q^{k} H_{k, 0} H_{n-k, 0}-\left(H_{n-1,0}+\sum_{k=1}^{n-2} q^{1+k} H_{k, 0} H_{n-1-k, 0}\right)\right) .
\end{aligned}
$$

Using the recurrence hypothesis for $H_{n, 1}$, we obtain

$$
\begin{aligned}
H_{n, 2} & =q\left(H_{n, 0}+\sum_{k=2}^{n-1} q^{k} H_{k, 0} H_{n-k, 0}-\left(H_{n, 1}-q H_{0,0} H_{n-1,0}\right)\right) \\
& =q\left(H_{0,0} H_{n, 0}+q H_{1,0} H_{n-1,0}+\sum_{k=2}^{n-1} q^{k} H_{k, 0} H_{n-k, 0}-H_{n, 1}\right)\left(\text { since } H_{0,0}=H_{1,0}=1\right) \\
& =\sum_{k=0}^{n-1} q^{1+k} H_{k, 0} H_{n-k, 0}-q H_{n, 1} .
\end{aligned}
$$

Thus, when returning to (18), we obtain

$$
\begin{aligned}
H_{n+1,1} & =H_{n, 0}+q H_{n, 1}+\sum_{k=0}^{n-1} q^{1+k} H_{k, 0} H_{n-k, 0}-q H_{n, 1} \\
& =H_{n, 0}+\sum_{k=0}^{n-1} q^{1+k} H_{k, 0} H_{n-k, 0} .
\end{aligned}
$$


Now, we can treat by the same way the case $i \geqslant 2$ as follows. We start from the relation

$$
H_{n+1, i}=q^{i-1} H_{n, i-1}+q^{i} H_{n, i}+H_{n, i+1} .
$$

Then, using (17) for $H_{n, i+1}$, we get

$$
H_{n, i+1}=q^{i-1}\left(H_{n-1, i}+\sum_{k=i}^{n-2} q^{1+k} H_{k, i} H_{n-1-k, 0}\right) .
$$

Now, replace $H_{k, i}$ by $H_{k+1, i-1}-q^{i-2} H_{k, i-2}-q^{i-1} H_{k, i-1}$ for any integer $i \geqslant 2$ and manipulate the obtained identity to get

$$
\begin{aligned}
H_{n, i+1} & =q^{i}(H_{n, i-1}+\sum_{k+1}^{n-1} q^{k} H_{k, i-1} H_{n-k, 0}-\underbrace{q^{i-2}\left(H_{n-1, i-2}+\sum_{k=i}^{n-2} q^{1+k} H_{k, i-2} H_{n-1-k, 0}\right)}_{A} \\
& -\underbrace{q^{i}\left(H_{n-1, i-1}+\sum_{k=i}^{n-2} q^{1+k} H_{k, i-1} H_{n-1-k, 0}\right)}_{B})
\end{aligned}
$$

Using the recurrence hypothesis for $H_{n, i-1}$ and $H_{n, i}$, we get

$$
\begin{aligned}
A & =H_{n, i-1}-q^{i-2}\left(q^{i-1} H_{i-2, i-2} H_{n+1-i, 0}+q^{i} H_{i-1, i-2} H_{n-i, 0}\right) \\
& =H_{n, i-1}-q^{i-2}\left(H_{i-1, i-1} H_{n+1-i, 0}+q^{i} H_{i-1, i-2} H_{n-i, 0}\right) \text { since } q^{i-1} H_{i-2, i-2}=H_{i-1, i-1} .
\end{aligned}
$$

and $B=H_{n, i}+q^{i-1}\left(q^{i} H_{i-1, i-1} H_{n-i, 0}\right)$. Thus, using also the fact that $q^{i-2} H_{i-1, i-2}+$ $q^{i-1} H_{i-1, i-1}=H_{i, i-1}$, we get

$$
A+B=H_{n, i}+H_{n, i-1}-q^{i-2} H_{i-1, i-1} H_{n+1-i, 0}-q^{i} H_{i, i-1} H_{n-i, 0} .
$$

Consequently, we obtain

$$
\begin{aligned}
H_{n, i+1} & =q^{i}\left(q^{i-2} H_{i-1, i-1} H_{n+1-i, 0}+q^{i} H_{i, i-1} H_{n-i, 0}+\sum_{k=i+1}^{n-1} q^{k} H_{k, i-1} H_{n-k, 0}-H_{n, i}\right) \\
& =q^{i}\left(\sum_{k=i-1}^{n-1} q^{k} H_{k, i-1} H_{n-k, 0}-H_{n, i}\right) \\
& =q^{i-1} \sum_{k=i-1}^{n-1} q^{1+k} H_{k, i-1} H_{n-k, 0}-q^{i} H_{n, i} .
\end{aligned}
$$

So, when returning to (19) and substitute $H_{n, i+1}$, we obtain

$$
H_{n+1, i}=q^{i-1}\left(H_{n, i-1}+\sum_{k=i-1}^{n-1} q^{1+k} H_{k, i-1} H_{n-k, 0}\right) .
$$

This also ends the proof of Theorem 14 . 
Corollary 15. For any positive integer $n$, we have $H_{n, 0}=\tilde{M}_{n}(q)$.

Proof. Using (15) with the fact that $H_{n, 0}=H_{n-1,0}+H_{n-1,1}$ for any integer $n \geqslant 0$, we get

$$
\begin{aligned}
H_{n, 0} & =H_{n-1,0}+H_{n-2,0}+\sum_{k=0}^{n-3} q^{1+k} H_{k, 0} H_{n-1-k, 0} \\
& =H_{n-1,0}+\sum_{k=0}^{n-2} q^{1+k-(n-1) \delta_{k, n-2}} H_{k, 0} H_{n-1-k, 0} .
\end{aligned}
$$

Since $H_{n, 0}$ and $\tilde{M}_{n}(q)$ satisfy the same recursion (see (5)) and the same initial values, then they are equal.

Combining this last corollary with identity (16), we obtain our desired result.

Theorem 16. For any positive integer n, we have

$$
\sum_{\sigma \in S_{n}(321,3 \overline{1} 42)} q^{\mathrm{cr}(\sigma)}=\tilde{M}_{n}(q) .
$$

We close this section with an interesting non-trivial identity concerning equality of two continued fractions as another consequence of Corollary 15.

Theorem 17. We have the following identity

$$
\frac{1}{1-\frac{t+t^{2}}{1-\frac{q t^{2}}{1-\frac{q t+(q t)^{2}}{1-\frac{q^{3} t^{2}}{\ddots}}}}}=\frac{1}{1-t-\frac{t^{2}}{1-q t-\frac{q t^{2}}{1-q^{2} t-\frac{q^{2} t^{2}}{\ddots}}}}
$$

Proof. Since the polynomial $\tilde{M}_{n}(q)$ satisfies the following recursion

$$
\tilde{M}_{0}(q)=1, \tilde{M}_{n}(q)=\tilde{M}_{n-1}(q)+\tilde{M}_{n-2}(q)+\sum_{k=0}^{n-3} q^{1+k} \tilde{M}_{k}(q) \tilde{M}_{n-2-k}(q) \text { for } n \geqslant 1,
$$

then its generating function, $\tilde{M}(q ; t):=\sum_{n \geqslant 0} \tilde{M}_{n}(q) t^{n}$, satisfies

$$
\tilde{M}(q ; t)=\frac{1}{1-\frac{t+t^{2}}{1-q t^{2} \tilde{M}(q ; q t)}} .
$$


So, when we continue to develop $\tilde{M}\left(q ; q^{k} t\right)$ for $k \geqslant 1$, we obtain the continued fraction expansion for $\tilde{M}(q ; t)$

$$
\tilde{M}(q ; t)=\frac{1}{1-\frac{t+t^{2}}{1-\frac{q t^{2}}{1-\frac{q t+(q t)^{2}}{1-\frac{q^{3} t^{2}}{\ddots}}}} .}
$$

Combining Corollary 15 with identity (16), we also have

$$
\tilde{M}(q ; t)=\frac{1}{1-t-\frac{t^{2}}{1-q t-\frac{q t^{2}}{1-q^{2} t-\frac{q^{2} t^{2}}{\ddots}}} .}
$$

This proves the desired identity of our theorem.

\section{Acknowledgments}

We would like to thank Arthur Randrianarivony who never accepted that we state Theorem 16 as a conjecture. A helpful discussion with him allowed us to find the recursion (17) of Theorem 14.

\section{References}

[1] E. Barcucci, A. Del Lungo, J.M. Fédou, R. Pinzani, Steep polyominoes, q-Motzkin numbers and q-Bessel functions, Discrete Math. 189 (1998) 21-42.

[2] M. Barnabei, F. Bonetti, M. Silimbani, Restricted involutions and Motzkin paths, Adv. Appl. Math. 47 (2011) 102-115.

[3] M. Barnabei, F. Bonetti, N. Castronuovo, and M. Silimbani, Motzkin and Catalan Tunnel Polynomials, J. Integer Sequences 21 (2018), Article 18.8.8.

[4] P. Biane, Permutations suivant le type d'excédance et le nombre d'inversions et interprétation combinatoire d'une fraction continue de Heine, European J. Combin. 14 (1993) 277-284.

[5] S. Burrill, M. Mishna, and J. Post, On k-crossing and k-nesting of permutation, DMTCS proc.AN (2010) 593-600.

[6] William Y. C. Chen, Yu-Ping Deng, and Laura L. M. Yang, Motzkin Paths and Reduced Decompositions for Permutations with Forbidden Patterns, Electron. J. Combin. 9(2) (2003),\#R15. 
[7] S. Corteel, Crossing and alignments of permutations, Adv. Appl. Math. 38:2 (2007) 149-163.

[8] S. Corteel, M. Josuat-Vergès, and J. S. Kim, Crossings of signed permutations and q-Eulerian numbers of type B, J. Comb. 4 (2013) 191-228.

[9] D. Dumont, Fractions continues et chemin de Motzkins, Support de cours Master 2, Université d'Antananarivo, 1993.

[10] D. Dumont and A. Randrianarivony, Sur une Extension des Nombres de Genocchi, European J. Combin. (1995) 16, 147-151.

[11] Eric S. Egge, Restricted 3412-avoiding involutions, continued fractions, and Chebyshev polynomials, Adv. Appl. Math. 33 (2004) 451-475.

[12] S. Elizalde and E. Deutsch, A Simple and Unusual Bijection for Dyck Paths and its Consequences, Ann. Comb. 7 (2003) 281-297.

[13] S. Elizalde and I. Pak, Bijections for refined restricted permutations, J. Combin. Theory Ser. A 105 (2004) 207-219.

[14] S. Elizalde, Multiple pattern avoidance with respect to fixed points and excedances, Electron. J. Combin. 11(1)(2004), \#R51.

[15] S. Elizalde, Continued fractions for permutation statistics, Discrete Math. Theor. Comput. Sci. 19(2)(2018), \#11.

[16] P. Flajolet, Combinatorial aspects of continued fractions, Discrete. Math. 32 (1980), pp. $125-161$.

[17] A. de Médicis and X.G. Viennot, Moments des q-polynômes de Laguerre et la bijection de Foata-Zeilberger, Adv. Appl. Math. 15 (1994) 262-304.

[18] P. M. Rakotomamonjy, Restricted permutations refined by number of crossings and nestings, Discrete Math. 343 (2020) 111950.

[19] P. M. Rakotomamonjy, S. R. Andriantsoa, and A. Randrianarivony, Crossings over Permutations Avoiding Some Pairs of Patterns of Length Three, J. Integer Sequences 23 (2020), Article 20.6.3.

[20] A. Randrianarivony, Fractions Continues, q-Nombres de Catalan et q-Polynômes de Genocchi, European J. Combin. 18 (1997), 75-92.

[21] A. Randrianarivony, q,p-analogues des nombres de Catalan, Discrete Math. 178 (1998), 199-211.

[22] N. J. A. Sloane, The Online Encyclopedia of Integer Sequences, published electronically at https://oeis.org, 2019. 